Cahiers de recherches médiévales

\title{
Réception de la littérature médiévale à travers le médium cinématographique
}

William Lucas

\section{(2) OpenEdition \\ 1 Journals}

Édition électronique

URL : https://journals.openedition.org/crm/2497

DOI : $10.4000 / \mathrm{crm} .2497$

ISSN : 1955-2424

Éditeur

Honoré Champion

Édition imprimée

Date de publication : 12 décembre 1996

Pagination : 149-153

ISSN : $1272-9752$

Référence électronique

William Lucas, « Réception de la littérature médiévale à travers le médium cinématographique »,

Cahiers de recherches médiévales [En ligne], 2 | 1996, mis en ligne le 04 février 2008, consulté le 15

décembre 2022. URL : http://journals.openedition.org/crm/2497 ; DOI : https://doi.org/10.4000/crm. 2497 


\section{RM}

\section{Réception de la littérature médiévale à travers le médium cinématographique}

Le cinéma s'est très tôt intéressé au Moyen Age, dès 1910 précisément avec la sortie sur les écrans américains du Lancelot et Elaine de James Stuart Backton. Alors que celui que l'on a coutume de nommer le septième art vient de fêter son premier centenaire, il paraît nécessaire d'établir, non pas un bilan, mais plutôt une mise au point.

Qu'est-ce que le cinéma? A quoi sert-il? A divertir répondra-t-on dans un premier temps, c'est vrai, mais à réfléchir aussi. Cohabitent ainsi difficilement deux types d'œurves: le film de «divertissement », bénéficiant le plus souvent d'un budget confortable, et ce que l'on appelle par convention «le film d'auteur ». Dans les deux cas, il s'agit de rencontrer le succès, commercial ou symbolique. Cette bipartition, quelque peu sommaire convenons-en, remonte aux origines du cinema puisque the Story of the Kelly Gang, datant de 1906, est considéré comme le premier grand divertissement cinématographique tandis que l'Assassinat du duc de Guise, sorti deux ans plus tard, passe pour la première œuvre d'art sur grand écran. Ainsi, Lancelot (U.S.A., 1995) de Jerry Zucker et Perceval le Gallois (France, 1979) d'Eric Rohmer ne semblent pas s'adresser au même public. A deux publics cinématographiques correspondent fort logiquement deux conceptions du Moyen Age. La première, liée à la grande majorité des spectateurs, inspire les films "commerciaux " et correspond à certaines images qui sont autant de clichés: des chevaliers, quelques châteauxforts, des rois et une épée (magique si possible). Il s'agit du Moyen Age que les enfants, particulièrement les jeunes américains, découvrent dès leur plus jeune âge à travers des bandes dessinées, des séries télévisées. Rappelons que l'univers médiéval, particulièrement la légende arthurienne, est exploitée à outrance aux Etats-Unis. La seconde conception du Moyen Age est celle qui nous est directement transmise par les textes médiévaux. Elle n'est en général que l'apanage de quelques universitaires chevronnés, et cela même si, grâce à des éditions comme celle des Lettres Gothiques, ce Moyen Age de conception classique tend actuellement à s'ouvrir à un public plus large.

Nous voici donc confrontés à deux conceptions de l'univers médiéval a priori peu compatibles. Ces deux «visions» ont-elles tendance à interférer l'une sur l'autre? Quelles sont les armes du cinéma d'auteur face au film commercial? Qu'est-ce finalement qu'adapter le Moyen Age au cinéma?

Ce que nous appelons pour simplifier le « film commercial » bénéficie en règle générale d'un budget conséquent, ce qui, paradoxalement, limite considérablement les possibilités du metteur en scène. En effet, la mise investie par le producteur doit être récupérée avec si possible du bénéfice. Or, malgré un siècle d'expérience, « les recettes du succès demeurent mystérieuses »', il s'agit donc de ne pas prendre de risques inutiles, afin de ne pas perdre la somme initialement investie. Plus le budget est important, plus la marge de créativité laissée au cinéaste est réduite. Les producteurs tendent donc, afin de prévenir un éventuel échec,

\footnotetext{
${ }^{1} \mathrm{~S}$. Tiaulier, Analyse de l'activité cinématographique par la théorie de l'innovation, Bibliothèque universitaire d'Orléans, D.EA. 1992/93, p. 4
} 
d'appliquer certaines recettes connues et reconnues pour leur efficacité: stars au générique, mise en scène spectaculaire (on privilégie ainsi d'habiles techniciens plutôt que de réels auteurs) et effets spéciaux novateurs. Cette recette, qui sans être infaillible a de bonnes chances d'être efficace, est particulièrement appropriée aux films inspirés par le Moyen Age. En effet, pour un producteur, qui dit « Moyen Age » dit «merveilleux », ce qui nécessite des effets spéciaux, et qui dit «effets spéciaux" dit «déplacement des foules», autrement dit «dollars». Considérons ainsi la dernière sortie des productions américaines sur le sujet, Dragonheart. Réalisé à la demande d'Universal par Rob Cohen, le film bénéficie d'un budget de soixante millions de dollars, d'effets spéciaux réalisés par I.L.M. (la firme de Georges Lucas) de Dennis Quaid dans le rôle principal et de Sean Connery, lequel prête sa voix au dragon. Ayons à l'esprit que pour le spectateur habitué à voir ce genre de produits, lesquels, en raison de grandes facilités de distribution, sont devenus majoritaires sur nos écrans, c'est cela le Moyen Age: des chevaliers, de la magie, des monstres. L'univers médiéval est tout simplement devenu de l'Héroïc Fantasy. Pour un producteur, Amold Schwarzenegger est aujourd'hui plus crédible dans le rôle de Perceval que, au hasard, Fabrice Luchini. Le merveilleux issu de nos romans courtois a été « traduit» par le biais des effets spéciaux, lesquels tendent de plus en plus à devenir l'intérêt principal du film.

Le cinéma que l'on a coutume d'appeler «d'auteur» ne s'adresse pas, nous l'avons vu dès l'introduction, au même public. Il n'a pas les contraintes liées à un gros budget, le capital investi étant surtout symbolique ${ }^{2}$, mais il n'en a pas non plus les avantages. Ses moyens de distribution, son support publicitaire, sont quasiment nuls. Le cinéaste, financièrement limité mais artistiquement libre, ne peut espérer concurrencer les productions commerciales et doit donc s'engager sur une autre voie. C'est ce qu'a fait dès 1979 Eric Rohmer avec son Perceval le Gallois. Le texte est composé de vers en octosyllabes, parlés ou chantés, au risque de désorienter le public (ce qui ne manqua pas d'arriver lors de la sortie du film en salle). Les décors, entièrement artificiels, évoquent les miniatures d'époque et le film luimême est chargé d'une signification qu'il serait vain de vouloir étudier dans un si bref exposé. L'auteur a visiblement cherché à s'éloigner du visuel médiéval habituellement présenté au cinéma. Plus proche de nous peut-être, et ne visant pas un simple capital symbolique, Terry Gilliam a été confronté avec Fisher King (U.S.A., 1990) au même type de problème. Bénéficiant d'un budget confortable et de stars au générique (Jeff Bridges et surtout Robin Williams), mais désireux de réaliser une véritable cuvre d'art (il n'est pas lieu ici de débattre sur ce qu'est une "véritable œuvre d'art »), l'auteur a dû abandonner toute idée de décor médiéval afin de ne pas avoir à souffrir la comparaison avec l'Héroï Fantasy qui, en raison des Barbarians et autres Kalidor, tend de plus en plus à devenir de l'Héroïc Fantaisiste.

Un film comme Excalibur (John Boorman, U.S.A., 1981) incarne les deux tendances actuelles du cinéma. Boorman flirte en effet avec le cinéma d'auteur, notamment grâce à ses études approfondies sur la symbolique, tout en réalisant presque un film d'Héroïc Fantasy (son Merlin semble être sorti des meilleures pages de Robert Howard) à une époque où ce genre cinématographique était particulièrement apprécié. Excalibur s'adresse dès lors au

\footnotetext{
${ }^{2}$ Concernant ces notions de "capital symbolique", on consultera le $n^{\circ} 13$ de la revue Actes de la recherche en sciences sociales, revue dirigée par Pierre Bourdieu et datée de février 1977.
} 
non-spécialiste pour qui nos héros restent souvent situés à la lisière de la fiction et de l'histoire, tout comme à l'amateur, soucieux de savoir ce qu'a pu devenir la matière de Bretagne concassée par la machine hollywoodienne.

Il est inévitable que, en raison d'une surexploitation, le Moyen Age ait perdu une bonne partie de sa crédibilité au cinéma. Des chansons de geste, les producteurs et scénaristes ont surtout retenu le schéma du héros isolé face à des « vilains » nettement supérieurs en nombre. Cette conception du Moyen Age semble avoir été remplacée à l'écran par le Western. Ce genre cinématographique, presque aussi vieux que le cinéma lui-même (le Vol du rapide, réalisé par Edwin S.Porter et produit par Edison date tout de même de 1903) a été relancé en 1990 par Kevin Costner avec Danse avec les loups (U.S.A.) et surtout par Clint Eastwood et son Impitoyable (U.S.A., 1992). Fleurissent alors sur les écrans des films comme Géronimo (Walter Hill, U.S.A., 1993), Tombstone (Georges Pan Cosmatos, U.S.A., 1993), Wyatt Earp (Lawrence Kashdan, U.S.A., 1994), les Belles de l'Ouest (Jonathan Kaplan, U.S.A., 1994), Mort ou vif (Sam Raimi, U.S.A., 1995) et, dans une moindre mesure Dead Man de Jim Jarmush (U.S.A., 1995). On peut aller jusqu'à affirmer qu'un film comme Waterworld (Kevin Reynolds, U.S.A., 1995) exploitant le même type de situation, est lui aussi un avatar cinématographique de la chanson de geste. Sans doute doit-on attribuer à cette recrudescence du Western la rareté des productions issues de l'univers du Roland. Considérons également qu'un cinéaste s'intéressant à la matière de Bretagne mais désireux d'occulter tout merveilleux ne réalise rien d'autre qu'un Western médiéval : preuve en est le Lancelot de Zucker.

Le cinéma a-t-il épuisé le Moyen Age? Intéressons-nous un instant au problème de la parodie. Parodier un genre peut signifier deux choses: soit que l'hypotexte (le parodié) ne présente plus le moindre intérêt, soit que l'hypertexte (le parodiant) est un hommage, « un processus sémiotique qui permet au récepteur de revoir les notions, les mots, les textes qu'il croit déjà connaître " ${ }^{3}$. Que penser de Sacré Graal et de Fisher King, tous deux réalisés, à seize ans d'intervalle, par Terry Gilliam? Le film des Monty Pythons, assez peu ambigu, repose sur une connaissance solide de la légende arthurienne et avoue des intentions pédagogiques que n'aurait pas reniées Eric Rohmer. Il s'agit, à n'en pas douter, d'un hommage respectueux. Fisher King au contraire se moque ouvertement des éléments directement tirés de la légende arthurienne, du Graal notamment, "machin genre verre à jus de fruit de Jésus» selon Anne (il ne s'agit que d'une traduction). Pour cet auteur (tout comme pour Rohmer), les décors et éléments issus des textes médiévaux ont perdu de leur crédibilité. Fisher King est ainsi délibérément situé dans le New-York des années 90.

Mais qu'est-ce qu'adapter le Moyen Age au cinéma ? S'agit-il d'adapter un ou plusieurs romans? Rien d'étonnant dès lors à ce que l'œuvre de base subisse, comme c'est le cas chez Rohmer ou Boorman, de nombreuses modifications. Le cinéma est un médium à temps limité ; nécessitant une composition synthétique, le roman au contraire ignore les limites du temps et de l'espace. Etienne Fuzellier précise que « le cinéaste procède en grande partie

${ }^{3}$ Anthony Wall, «Vers une notion de la colle parodique ", article paru dans Etudes littéraires volume $19 n^{\circ} 1$, université de Lanval, printempsété 1986, p. 26. 
comme un dramaturge qui voudrait, de ce roman, tirer une pièce de théâtre " ${ }^{4}$. Dans une œuvre littéraire, l'espace diégétique est dit, alors qu'au cinéma, il est montré et éventuellement accompagné d'un fond sonore et d'une évocation ou description verbale. II ne s'agit tout simplement pas du même langage, le cinéaste doit choisir entre «l'attitude respectueuse et l'attitude créatrice ${ }^{5}$. Mais, nous l'avons précisé dès notre introduction, les textes sont finalement assez peu connus du grand public. Nous devrions donc, afin d'être plus précis, nous contenter de parler de réminiscences, de certains topoï que le lecteur occasionnel aurait retenus, de certains schémas que le cinéaste aurait à réutiliser. Parmi les situations-types (c'est à dessein que nous nous refusons à employer le terme péjoratif de "cliché »), il semble que ce soit celle du héros, ou d'un groupe de personnages, subordonné à une quête que nos contemporains retiennent le mieux. Considérons à ce propos une nouvelle fois Fisher King ou Terry Gilliam illustre le motif de la quête intérieure, cher à Lancelot et Perceval. Jack doit en effet transcender son personnage afin de vivre en paix, il s'agit de retrouver la foi en soi-même, de dépasser son propre système de valeur (l'argent), de trouver le Graal. Le calice en lui-même n'est rien, seule compte la motivation du personnage qui, pour la première fois, n'agit pas pour lui-même. Tout comme dans la Quête $d u$ Graal, l'aventure dans Fisher King "se manifeste moins par une suite d'exploits brillants (...) que par une écoute aux voix qui sollicitent une réforme profonde et une purification grâce à laquelle se libère l'esprit » ${ }^{6}$. De même, les héros de la trilogie de la Guerne des Etoiles, fortement inspirée par la légende arthurienne aux dires de Georges Lucas lui-même, sont subordonnés au motif de la quête : quête de la Force, du père, de la liberté, quête de soimême surtout.

Le cinéaste désireux de réaliser un film inspiré de l'univers médiéval semble également être obligé de nourrir son œuvre de symbolisme. Du Chevalier Rouge cracheur de feu de Fisher King à la voûte de Perceval le Gallois, du symbolisme des couleurs de Boorman à celui de Georges Lucas, l'image devient signifiant et le film un univers pluricodé. Tous cependant ne présentent pas la même difficulté. Le symbolisme d'Indiana Jones et la Dernière Croisade (Steven Spielberg, U.S.A., 1989) est on ne peut plus simple: lors de la scène finale, l'humilité du héros choisissant le calice de bois s'oppose à l'avidité du nazi préférant l'or. Celui de Perceval le Gallois nécessite en revanche les volumineuses études de Joseph Marty qui elles-mêmes, demandent plusieurs lectures. Quoiqu'il en soit, cette recherche du symbole semble être une constante difficile à expliquer. Sans doute faut-il une nouvelle fois recourir à la bipartition qui préside à notre exposé afin de fournir une tentative d'explication. Le cinéaste commercial cherche, par le biais du symbolisme, à gagner son statut d'auteur, souvent peu compatible avec des préoccupations mercantiles mais indiciblement lié à la littérature médiévale, réservée le plus souvent à l'étude. Quant au cinéaste déjà reconnu comme auteur, il peut, grâce au symbolisme, conférer à son œuvre une atmosphère «étrange ", traduction cinématographique du merveilleux littéraire, sans pour autant recourir aux effets spéciaux, évitant ainsi d'apparenter son film à de l'Hérö̈c Fantasy.

\footnotetext{
${ }^{4}$ Etienne Fuzellier, Cinéma et littérature, Paris, 1964, Collection septième art, Editions du Cerf, p. 68

${ }^{5}$ Ibid, p. 130.

${ }^{6}$ Alexandre Micha, Essais sur le Lancelot-Graal, Genève, 1987, librairie Droz, p. 155.
} 
Que reste-t-il du Moyen Age aujourd'hui au cinéma? Les films d'auteur, malgré leur volonté de se démarquer, ont été littéralement absorbés par les entreprises commerciales. Perceval le Gallois date tout de même de 1979 et la demière tentative française dans le domaine les Chevaliers de la Table Ronde (Denis Llorca, 1990) a été un échec artistique autant que commercial. Parallèlement, Dragonheart est sorti l'été dernier sur les écrans américains et la Warner annonce le dessin animé Quest for Camelot. Les diverses exploitations ont privé de toute crédibilité les héros issus du Moyen Age. Paradoxalement, c'est peut-être d'Hollywood que viendra l'œuvre qui saura redonner ses lettres de noblesse au cinéma d'inspiration médiévale, du moins en ce qui concerne l'univers de la chanson de geste. Paul Verhoeven (Basic Instinct, Robocop, mais aussi et surtout la Chair et le Sang avec Rutger Hauer) annonce en effet régulièrement son Crusades avec Amold Schwarzenegger. Le film sera brutal, excessivement violent sans doute, mais permettra au spectateur de se familiariser avec l'univers du Roland. Un retour aux sources sans concession permettra peut-être d'ouvrir de nouvelles voies. Le merveilleux et la légende arthurienne ont quant à eux été dignement repris dès 1977 par Georges Lucas à travers sa trilogie de la Guerre des Etoiles. La matière de Bretagne, loin d'être une simple histoire de chevaliers, est une leçon d'humanité, de dépassement de soi. Elle ouvre aussi à l'imaginaire, tendant à rendre l'impossible possible, le rêve, réalité. Georges Lucas, avec respect, reprend cette leçon d'humanité et perpétue le rêve?

William Lucas

${ }^{7}$ En plus des ouvrages cités en notes, on pourra consulter :

Avant scène nं 221, 1979

Les Cahiers de la cinémathèque, revue d'histoire du cinéma éditée par l'institut Jean Vigo, $\mathrm{n}^{\circ} 42$ 43, êté 1985

Cinéma $1979, \mathrm{n}^{\circ} 242$

Eudes Cinématographiques n 149-152, septembre 1986

André Gardies, le Récit filmique, collection Contours littéraires, Hachette, Paris, 1993 